\title{
The Amyloid Precursor Protein and Its Regulatory Protein, FE65, in Growth Cones and Synapses In Vitro and In Vivo
}

\author{
Shasta L. Sabo, ${ }^{1}$ Annat F. Ikin, ${ }^{1,2}$ Joseph D. Buxbaum, ${ }^{2}$ and Paul Greengard ${ }^{1}$ \\ ${ }^{1}$ Laboratory of Molecular and Cellular Neuroscience and the Zachary and Elizabeth M. Fisher Center, The Rockefeller University, New York, New York \\ 10021, and 2Laboratory of Molecular Neuropsychiatry, Departments of Psychiatry and Neurobiology, Mount Sinai School of Medicine, New York, New York \\ 10029
}

\begin{abstract}
Although the Alzheimer amyloid protein precursor (APP) has been studied intensely for more than a decade, its function in neurons is unresolved. Much less is known about its binding partner FE65. We have shown recently that APP and FE65 synergistically regulate the movement of transfected cells. It remained to be shown whether endogenous APP and FE65 could play a similar role in vivo. Here, we show that FE65, like APP, is expressed at high levels in neurons. Using a combination of immunofluorescence, live imaging, and subcellular fractionation, we find that FE65 and APP localize in vitro and in vivo to the most motile regions of neurons, the growth cones. Within growth cones, APP and FE65 concentrate in actin-rich lamellipodia. Finally, APP and FE65 interact in nerve terminals, where they associate with Rab5-containing synaptic organelles but not with synaptic vesicles. Our data are consistent with a role for the APP/FE65 complex in regulation of actin-based membrane motility in neurons, which could be important for highly dynamic processes such as neurite growth and synapse modification.
\end{abstract}

Key words: APP; FE65; Mena; growth cone; synapse; Alzheimer's disease

\section{Introduction}

Although the function of the Alzheimer amyloid protein precursor (APP) in neurons remains unclear, genetic manipulations of APP expression have suggested that APP has roles in synaptogenesis and synaptic plasticity. For example, overexpression of APP in Drosophila results in altered synaptic structure (Torroja et al., 1999). In addition, deletion of the APP gene from mice leads to deficits in learning and memory, impaired long-term potentiation (LTP), and decreased expression of synaptic markers (Dawson et al., 1999). The mechanism by which APP exerts these effects is unknown, but the similar effects of decreasing and increasing APP expression suggest that a balance between APP and some other factor might be required for proper APP function. This factor may be FE65, a cytosolic adapter that regulates the subcellular trafficking and proteolytic processing of APP (Guenette et al., 1999; Sabo et al., 1999).

We have shown recently that APP, in a complex with FE65, can regulate cell motility in transfected cells (Sabo et al., 2001). FE65 interacts simultaneously with APP and Mena (Sabo et al., 2001), which is required for normal neural development (Gertler et al., 1990; Lanier et al., 1999). Mena regulates axonal responses to repulsive cues (Bashaw et al., 2000) and is thought to exert its

Received Feb. 12, 2003; revised April 28, 2003; accepted April 28, 2003.

This work was supported by United States Public Health Service Grants AG09464 (P.G.) and AG14996 (J.D.B.), Alzheimer Association grants (J.D.B, A.F.I), National Institutes of Health Training Grant GM07524 (S.L.S.), and a Rockefeller University fellowship (S.L.S.). We also thank the Mount Sinai Hybridoma Shared Research Facility. We thank Drs. E. H. Koo and F. Gertler for antibodies and Dr. F. Benfenati for synaptic vesicles. We thank Drs. A. K. McAllister and N. Spitzer for advice on live imaging.

Correspondence should be addressed to Shasta L. Sabo, Center for Neuroscience, University of California, Davis, 1544 Newton Court, Davis, CA 95616. E-mail: slsabo@ucdavis.edu.

Copyright $\odot 2003$ Society for Neuroscience $\quad$ 0270-6474/03/235407-09\$15.00/0 effects by regulating actin dynamics in the growth cone. Together, these data suggest the hypothesis that the APP/FE65 complex regulates growth cone motility, possibly through its interaction with Mena.

If APP regulates growth cone motility and synaptic plasticity through its interaction with FE65, then it should interact with FE65 in growth cones and synapses. We show here that APP and FE65 colocalize in growth cones both in vitro and in vivo. We show further that APP and FE65 are found in actin-rich mobile structures within the growth cone, where they are poised to interact with the cytoskeleton indirectly through Mena. Finally, we provide evidence for an important interaction between APP and FE65 in synapses in vitro and in vivo as well. Our data are consistent with the hypothesis that APP and FE65 regulate membrane dynamics in growth cones and nerve terminals much as they do in lamellipodia of migrating cells.

\section{Materials and Methods}

Neuronal cell culture and transfection. Primary neuronal cultures were prepared from cortices of embryonic day (E) 17 or postnatal day (P) 1 rats. E17 brains were dissociated by trypsinization and trituration in DMEM with $10 \%$ fetal bovine serum. Dissociated neurons were cultured on poly-lysine or poly-lysine and laminin (Biocoat, Bedford MA) and maintained in neurobasal medium, B27 supplement (Invitrogen, Carlsbad CA), and antibiotics (Invitrogen). P1 brains were dissociated with papain and plated directly on glia in MEM, 10 mM HEPES, N2 supplement, glucose, and $10 \%$ horse serum.

Neurons were transfected 3 or $6 \mathrm{~d}$ after plating using Lipofectamine 2000 (Invitrogen) essentially according to the manufacturer's instructions. FE65-enhanced green fluorescent protein (EGFP) cDNA was made by subcloning FE65 into pEGFP-C1 (Clontech, Palo Alto CA). Synaptobrevin-dsRed was generated by subcloning synaptobrevin into 
pdsRed-N1 (Clontech). The localization of synaptobrevin-dsRed was identical to that of synaptobrevin-EGFP, which is correctly targeted to synaptic vesicles (Miesenbock et al., 1998; Nonet, 1999; Ahmari et al., 2000; Estes et al., 2000; Alsina et al., 2001).

Axons and dendrites were distinguished on the basis of accepted morphological criteria (Banker and Goslin, 1998). At early stages in culture, the axon is the longest process. Axons do not tend to taper. Conversely, dendrites are tapered and highly branched.

Antibodies. FE65 and APP (369) polyclonal antibodies were affinity purified as described (Sabo et al., 1999). FE65 monoclonal antibody (3H6) was raised against the WW domain of FE65. FE65 monoclonal and polyclonal antibodies label the same subcellular structures. APP monoclonal antibodies (5A3/1G7) and Mena antibodies were gifts from Drs. E. Koo (University of California San Diego) and F. Gertler (Massachusetts Institute of Technology), respectively. GAP-43 antibodies were purchased from Transduction Laboratories. Synaptophysin monoclonal antibody (C7.2) has been described previously (Jahn et al., 1985). Rab5 and synaptobrevin antibodies have been characterized previously (Fischer von Mollard et al., 1994; Chilcote et al., 1995).

Immunoblotting. Nitrocellulose membranes were blocked with Trisbuffered saline containing $5 \%(\mathrm{w} / \mathrm{v})$ nonfat dry milk and $0.05 \%$ Tween 20 (TBS-T) and then probed with primary antibodies and HRPconjugated secondary antibody (Calbiochem, La Jolla CA) in TBS-T. Immunoreactivity was visualized by ECL (DuPont NEN, Boston, MA) and exposure to $\mathrm{x}$-ray film (DuPont NEN).

Immunofluorescence. Cells were fixed in 4\% paraformaldehyde in PBS with $0.12 \mathrm{M}$ sucrose, permeabilized with $0.1 \%$ Triton X-100 in PBS, blocked with $10 \%$ normal goat serum (NGS) in PBS, and then immunolabeled in 5\% NGS in PBS. Coverslips were mounted with 1,4diazbicyclo (2.2.2) octane in Tris-buffered polyvinyl alcohol and glycerol. Secondary antibodies were goat anti-mouse Oregon Green, goat anti-rabbit Texas Red (Molecular Probes, Eugene OR), goat anti-mouse Cy5, and goat anti-rabbit Rhodamine red X (Jackson ImmunoResearch, West Grove PA). Actin was detected with Oregon Green phalloidin (Molecular Probes). All immunofluorescence was eliminated by omission of primary antibody. In multiple label experiments, labeling patterns were identical to those seen with single labeling, and channels were imaged sequentially to avoid bleed-through. Immunofluorescence was examined by confocal laser scanning microscopy (Zeiss LSM510 or Leica TCSSP) using $63 \times$ and $40 \times$ lenses, Ar488, KrNe543, and KrNe633 nm lasers, and BP560-615, BP505-550, and LP650 filters. For colocalization, optical sections were no thicker than $1.5 \mu \mathrm{m}$.

Live imaging. Neurons were transferred to imaging solution containing (in mM): $120 \mathrm{NaCl}, 3 \mathrm{KCl}, 2 \mathrm{CaCl}_{2}, 2 \mathrm{MgCl}_{2}, 30$ dextrose, 20 HEPES, pH 7.3 (McAllister and Stevens, 2000). During live imaging, neurons were kept at $29-32^{\circ} \mathrm{C}$ by constant perfusion with warmed imaging solution. The laser power was reduced to $5 \%$, and the inter-image interval was at least twice as long as the image collection time, minimizing phototoxicity. Under these conditions, filopodia were highly mobile, as were synaptobrevin-containing transport organelles, indicating that the neurons were healthy and active.

Image quantification. The mean pixel intensity for each image was determined in Photoshop 5.0. Each image was thresholded at the mean intensity plus 2 SDs above the mean. The thresholded images were then multiplied. At any pixel where intensity $=0$ (i.e., no fluorescence) for either image, the product $=0$; therefore, non-zero pixel intensities correspond to colocalization.

Growth cone fractionation. Growth cone particles (GCPs) were prepared as described previously (Pfenninger et al., 1983). E17-18 rat brains (20-30) were homogenized in $1 \mathrm{~mm} \mathrm{MgCl}_{2}, 0.32 \mathrm{M}$ sucrose, $1 \mathrm{~mm}$ $N$-[Tris(hydroxymethyl)methyl]-2-aminoethanesulfonic acid, $\mathrm{pH} 7.3$, and the $1660 \times g$ supernatant was separated on a 2.66 and $0.83 \mathrm{M}$ sucrose step gradient. GCPs were removed and washed with $0.32 \mathrm{M}$ sucrose buffer. Growth cone membranes (GCMs) were prepared by lysis of GCPs in hypotonic buffer ( 1 mM EDTA, 6 mM Tris, $\mathrm{pH}$ 8.1) with a tight-fitting glass-glass homogenizer and centrifugation at $200,000 \times g$. Equal protein from each fraction was analyzed by SDS-PAGE and immunoblotting.

Synaptosome fractionation. Synaptosomes and synaptic vesicles were prepared by standard methods (Huttner et al., 1983). Rat brains were
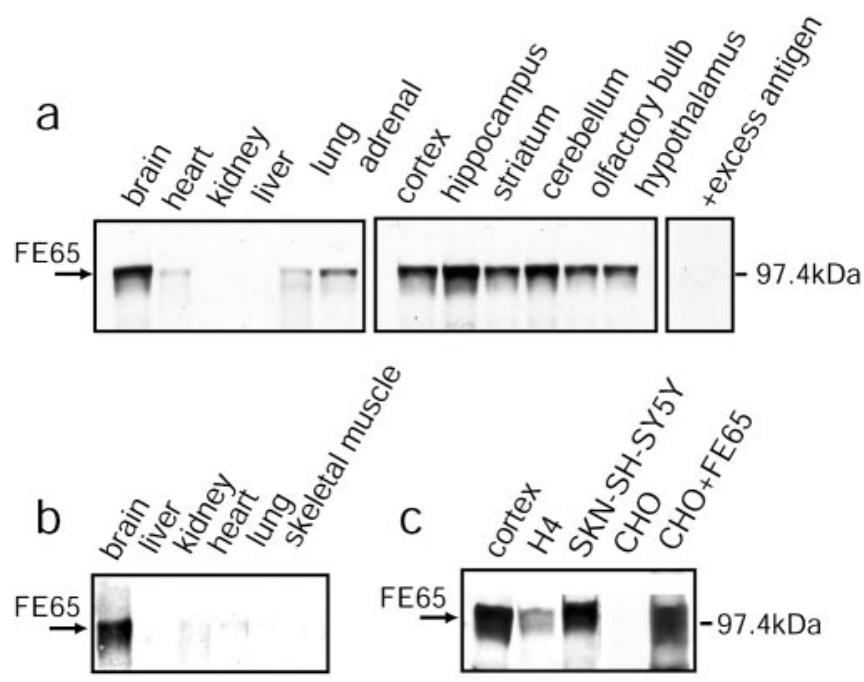

Figure 1. FE65 is highly enriched in brain. $a$, Tissues from adult rat were immunoblotted with FE65 antibody. Preincubation of FE65 antibody with excess antigen (+ excess antigen) eliminated the FE65-immunoreactive bands. $b$, Immunoblotting of human tissues for FE65. $c$ Immunoblotting of expressed FE65 in neural (SKN-SH-SY5Y, H4) and non-neural cell lines with (CHO+FE65) and without (CH0) FE65 transfection.

homogenized in $0.32 \mathrm{M}$ sucrose, $4 \mathrm{~mm}$ HEPES-NaOH, $\mathrm{pH}$ 7.3, with protease inhibitors. After centrifugation for $10 \mathrm{~min}$ at $1000 \times g$, the supernatant was centrifuged for $15 \mathrm{~min}$ at $12,000 \times \mathrm{g}$. The washed pellet is the crude synaptosomal fraction (P2). Fractions enriched in synaptic vesicles, obtained from Dr. F. Benfenati (University of Genova), were released from synaptosomes by hypotonic lysis and then isolated by centrifugation on a sucrose gradient.

Coprecipitation. Crude synaptosomes were suspended in 1\% Triton X-100, $50 \mathrm{~mm} \mathrm{NaPO}_{4}, 5 \mathrm{~mm}$ EDTA, protease inhibitors, and $20 \mathrm{~mm}$ HEPES, pH 7.4. After centrifugation at $40,000 \times g$, the supernatant was incubated with glutathione-Sepharose beads saturated with glutathione $S$-transferase (GST)-fusion protein. After extensive washing, bound proteins were analyzed by SDS-PAGE and immunoblotting.

Immunoisolation. Immunoisolation was performed as described (Ikin et al., 1996). IgG was purified on Protein G-Sepharose (Amersham Biosciences, Piscataway NJ) and then coupled to Eupergit C1Z beads (Rohm Pharma, Darmstadt, Germany). Synaptosomes were isolated using a combination of differential centrifugation (described above) and a threestep Ficoll gradient (12, 9, and 6\% Ficoll in $0.32 \mathrm{~m}$ sucrose). Purified synaptosomes were hypotonically lysed and then made isotonic. Centrifugation for $15 \mathrm{~min}$ at $18,700 \times \mathrm{g}$ separated the synaptic organelles from synaptosomal membrane debris. Synaptic organelles were incubated with immunobeads for $45 \mathrm{~min}$ at $4^{\circ} \mathrm{C}$ under slow rotation, and then beads were washed with $0.32 \mathrm{~m}$ sucrose, $10 \mathrm{~mm}$ HEPES, pH 7.4. Because of the IgG in the sample, equal amounts of synaptophysin, rather than total protein, were loaded on SDS-PAGE gels for comparison of the samples.

\section{Results}

\section{FE65 is enriched in human and rat brains}

It was important to establish first whether FE65 is expressed in neurons in vivo. Immunoblotting with FE65 antibodies showed that expression of FE65 $(\sim 100 \mathrm{kDa})$ was highest in the brains of rats and humans (Fig. 1a,b). Low levels of expression in some peripheral tissues might be caused by innervation. Expression was high throughout the brain and particularly high in cortex, hippocampus, and cerebellum (Fig. 1a). APP is also expressed in all brain areas, with cortex, hippocampus, and cerebellum among the areas expressing especially high levels of APP (Shivers et al., 1988).

FE65 antibodies also recognized a second band in brain, at 

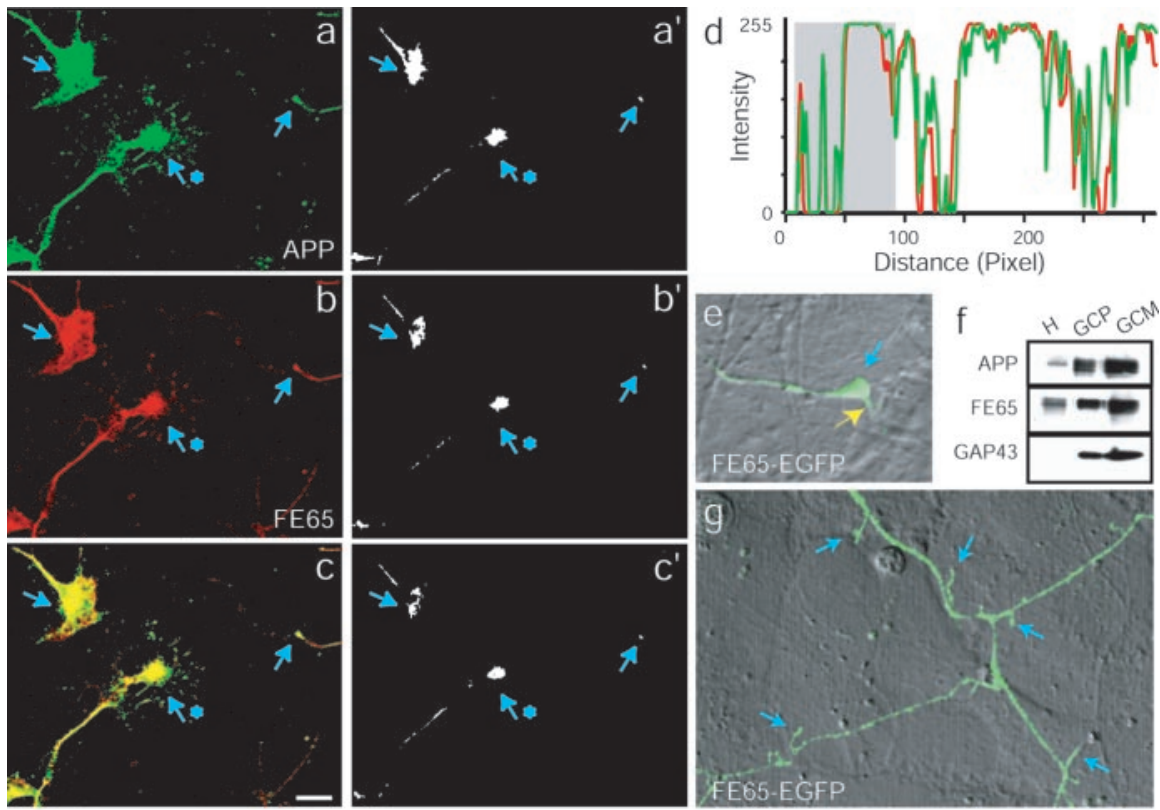

Figure 2. FE65 and APP localize to growth cones in vitro and in vivo. $a-c$, Confocal images of several embryonic neurona growth cones double-labeled with APP $(a)$ and FE65 $(b)$ antibodies. Yellow in the overlay $(c)$ represents colocalization of APP and FE65. Blue arrows point to the intense labeling of three growth cones by APP and FE65 antibodies. $a^{\prime}-c^{\prime}$, Thresholded versions of the images in $a$-c illustrate that the most intense labeling for both APP and FE65 (represented by white in the images) is found within growth cones (indicated by blue arrows). $d$, Line intensity profile of the intensity of APP (green) and FE65 (red) labeling in the growth cone and axon indicated by the blue star in $a-c$. The line was drawn through the center of the growth cone and down the axon, parallel to the axonal axis. The region that corresponds to the growth cone is indicated by a gray box in the intensity profile. Although intensity values reached saturation within the growth cone, intensity values were lower and highly variable within the axon. The close covariance of the red and green signals illustrates the high degree of colocalization of APP and FE65. The two sharp peaks at the beginning of the profile correspond to puncta of APP and FE65 within the growth cone filopodia.e, Growth cone of a neuron transfected with FE65-EGFP. The fluorescence image is showed as an overlay on the DIC image. A blue arrow indicates the position of the growth cone on the labeled axon. Notice the labeled filopodium on the bottom, right (shown with a yellow arrow). $f$, Immunoblotting of purified growth cones with APP, FE65, and GAP-43 antibodies. GAP-43 was used to monitor the purification procedure. $\mathrm{H}$, Embryonic rat brain homogenate; GCP, growth cone particles; GCM, growth cone membranes. $g$, Dendritic filopodia (indicated by blue arrows) of a neuron transfected with FE65-EGFP. The fluorescence image is shown as an overlay on the DIC image. Dendrites were identified by conventional morphological criteria (see Materials and Methods) (Banker and Goslin, 1998).

$\sim 60 \mathrm{kDa}$ (data not shown). We do not yet know the identity of the smaller band, which we refer to as FE65LP (for FE65-like protein). Both the FE65 and FE65LP bands disappeared after preincubation of the antibody with excess antigen (Fig. 1a). In addition, the same two bands were recognized by three additional FE65 antisera (data not shown). FE65LP was not seen in Chinese hamster ovary $(\mathrm{CHO})$ cells transfected with FE65 cDNA or in neural cell lines that express FE65, including SKN-SH-SY5Y human neuroblastoma cells and H4 human neuroglioma cells (Fig. 1c). FE65LP most likely corresponds to FE65L2, an FE65 homolog that has a predicted molecular weight of $55-60 \mathrm{kDa}$ (Duilio et al., 1998; Tanahashi and Tabira, 1999).

\section{FE65 and APP colocalize in neuronal growth cones in vitro and in vivo}

When primary neuronal cultures were examined by immunofluorescence, FE65 and APP colocalized in growth cones (Fig. 2a-d). Both proteins were found in growth cones with various morphologies and at the tips of both long and short neurites. In addition, labeling of neurons grown on laminin was indistinguishable from that of neurons grown on poly-lysine (data not shown). These results agree with reports citing the presence of APP in growth cones (Ferreira et al., 1993; Yamazaki et al., 1997). There was also labeling for APP and FE65 in the axon shaft, which is not surpris-

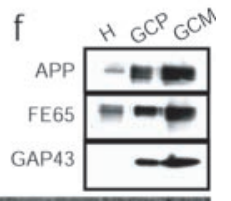

ing given that after synthesis APP and FE65 are most likely transported down the axon to the growth cone. Portions of the images in Figure $2 a-c$ appear saturated because intensity levels were elevated to reveal APP and FE65 in the very thin growth cone filopodia. To illustrate better the localization of APP and FE65 to growth cones, the images from Figure $2 a-c$ were thresholded, revealing only the most intense labeling (Fig. $2 a^{\prime}-c^{\prime}$ ). The thresholded images show that APP and FE65 are more intense in growth cones than in neighboring regions of the axon shaft. To further illustrate this point, a line intensity profile (for a line drawn parallel to the axon axis and through both the growth cone and the axon) is shown for one of the growth cones shown in the images (Fig. $2 d)$. The most intense labeling is found in the growth cone region. Together these data clearly show that the two proteins localize to growth cones.

To ensure that FE65 immunoreactivity in growth cones could not be attributed solely to FE65LP, we transfected cultured primary neurons with cDNA encoding FE65 fused to EGFP (FE65-EGFP). Live imaging of FE65-EGFP confirmed the localization of FE65 in growth cones (Fig. $2 e$ ). In addition, FE65 and APP colocalized in the growth cones of differentiated $\mathrm{SH}$ SY5Y cells, which do not express FE65LP (data not shown).

To determine whether APP and FE65 localize to growth cones in vivo, growth cones isolated from embryonic rat brain were probed for APP and FE65 by immunoblotting (Fig. 2f). These GCPs contain all proteins tested that are known to be in growth cones in vivo (Pfenninger et al., 1983). APP and FE65 both copurified with GCP and GCMs. Growth cone enrichment was confirmed by probing for GAP-43, which is concentrated in growth cones. Although FE65 is a soluble protein, it associated with GCM, possibly through its interaction with APP. These data suggest that the APP/FE65 complex might regulate growth cone motility in vivo, analogous to its role in regulation of cell movement.

Interestingly, FE65-EGFP also localized to dendritic shaft filopodia (Fig. $2 g$ ), highly mobile structures known to depend on actin dynamics for their motility. At this stage of development, dendrites can be identified on the basis of their length (i.e., the axon is much longer than the dendrites) and by their decreasing caliper with increasing length (Banker and Goslin, 1998). In contrast, axons tend to be of relatively constant diameter. These data are consistent with a role for the FE65/APP complex in regulation of actin-based motility in neurons at sites other than the growth cone as well.

\section{FE65 and APP concentrate in growth cone peripheral domains}

Growth cones can be divided into two functional domains: the central $(\mathrm{C})$ domain and the peripheral $(\mathrm{P})$ domain. The $\mathrm{C}$ domain is enriched in microtubules and organelles. The $\mathrm{P}$ domain is 

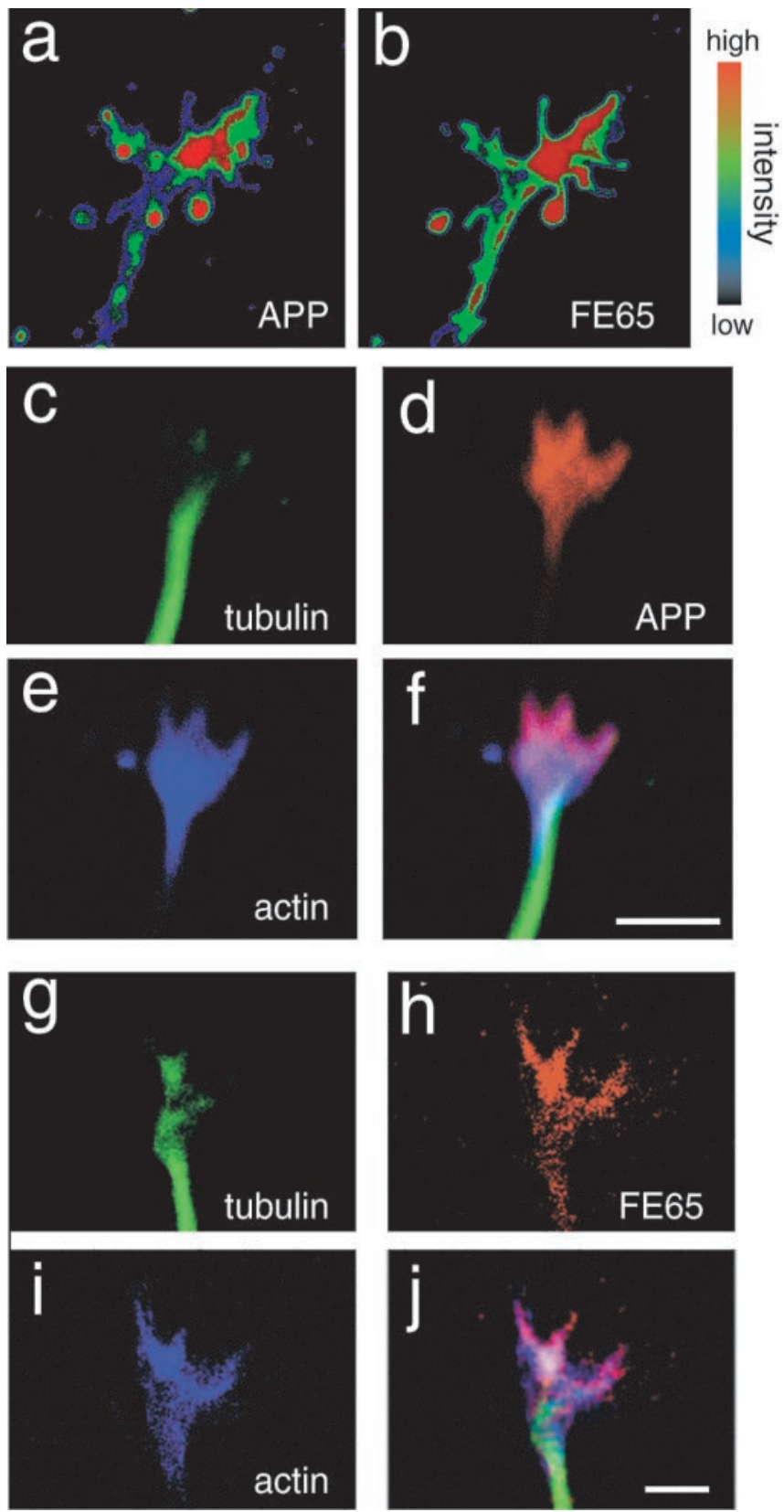

Figure 3. FE65 and APP localize to growth cone $P$ domains. Primary neuronal cultures from E17 rat brain were labeled with the indicated antibodies and stains and examined by confocal microscopy. a, b, Growth cone labeled with APP ( $a)$ and FE65 ( $b$ ) antibodies. Images were pseudocolored with an intensity scale indicated by the guide. Blue represents the lowest intensity, and red represents the highest intensity. The most intense staining was often in the more distal region of the growth cone. $c-f$, Growth cone labeled with tubulin antibody to label microtubules ( $c$ ) and with APP antibody ( $d$ ) and phalloidin to label F-actin (e). APP colocalizes more strongly with actin in the $P$ domain than with tubulin in the $($ domain. $g-j$, Growth cone labeled with tubulin antibody $(g)$, FE65 antibodies $(h)$, and phalloidin ( $i)$. FE65 also colocalizes more strongly with actin in the $P$ domain than with tubulin in the $C$ domain. Scale bars, $5 \mu \mathrm{m}$.

actin-rich, containing lamellipodia and filopodia, the most motile structures of the growth cone. If the APP/FE65 complex is involved in regulation of actin-dependent growth cone motility, then it should localize to the P domain.

Immunofluorescent labeling for APP and FE65 showed that both proteins often concentrated in distal regions of the growth cone, likely corresponding to $\mathrm{P}$ domain lamellipodia, and frequently extended into the base of the filopodia (Fig. $3 a, b$ ). Occa-
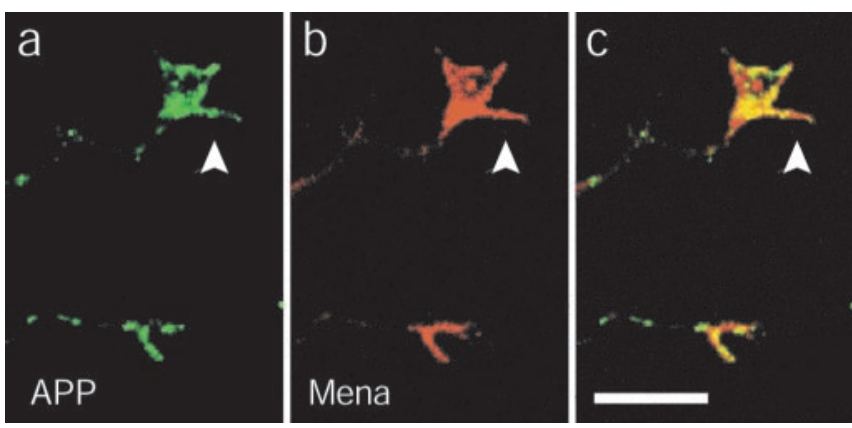

Figure 4. APP and Mena colocalize in growth cones. Primary neuronal cultures from E17 rat brain were double-labeled with APP $(a)$ and Mena $(b)$ antibody and examined by confocal microscopy. Arrows point at the growth cone in the image. Colocalization is indicated by yellow in the overlay (c).

sionally, staining was seen down the length of filopodia. FE65 and APP antibodies also recognized small puncta in the C domain that might correspond to transport vesicles being delivered to the growth cone because APP travels via fast axonal transport (Koo et al., 1990). When neurons were triple labeled with tubulin antibody to identify microtubules, Oregon Green-conjugated phalloidin to stain filamentous actin, and either APP or FE65 antibody, APP (Fig. 3c-f) and FE65 (Fig. 3g-j) immunofluorescence typically overlapped more with actin filaments than with microtubules.

Because APP and Mena colocalize in lamellipodia of motile cells (Sabo et al., 2001) and Mena is thought to regulate growth cone actin dynamics (Lanier et al., 1999), we tested whether APP and Mena might colocalize in growth cones. Double labeling of neurons with APP and Mena antibodies revealed colocalization of APP and Mena in growth cone lamellipodia (Fig. 4). These data are consistent with the idea that the FE65/APP complex regulates actin-based growth cone motility.

\section{APP and FE65 interact in nerve terminals}

In mature neurons, APP is enriched in nerve terminals (Koo et al., 1990; Yamazaki et al., 1995; Ikin et al., 1996). At 7-8 d in vitro (DIV), cortical cultures have established many stable synaptic contacts with neighboring neurons. To determine whether FE65, like APP, is found in nerve terminals, immunofluorescence of FE65, APP, and the synaptic vesicle protein synaptophysin was performed in neurons that had formed synapses. Synaptophysin staining was concentrated in discrete puncta, as described previously (Fig. 5b,f) (Fletcher et al., 1991), and looked like stereotypical presynaptic terminal staining with many presynaptic antibodies seen by many labs. Both APP (Fig. $5 a-c$ ) and FE65 (Fig. $5 e-g$ ) were found at sites that were enriched in synaptophysin. Because synaptophysin is a synaptic vesicle-associated protein, these sites are likely to be synapses.

To quantify the occurrence of FE65 and APP in synapses, the percentage of synaptophysin-positive synapses that also contained either FE65 or APP was determined. The fluorescence signals for APP (Fig. 5a) or FE65 (Fig. 5e) were thresholded and multiplied by the thresholded fluorescence signals for synaptophysin from the same neurons (Fig. $5 b, f$ ). The product of the two signals represents all sites in the image where either APP and synaptophysin (Fig. 5d) or FE65 and synaptophysin (Fig. 5h) colocalize. Of 191 synaptophysin-positive synapses counted along 9 randomly chosen axon segments, $67.6 \pm 4.0 \%$ (mean \pm $\mathrm{SE}$ ) of the synapses contained APP, and $84.4 \pm 5.0 \%$ (mean \pm $\mathrm{SE})$ of synaptophysin-positive synapses contained FE65 $(n=157$ 

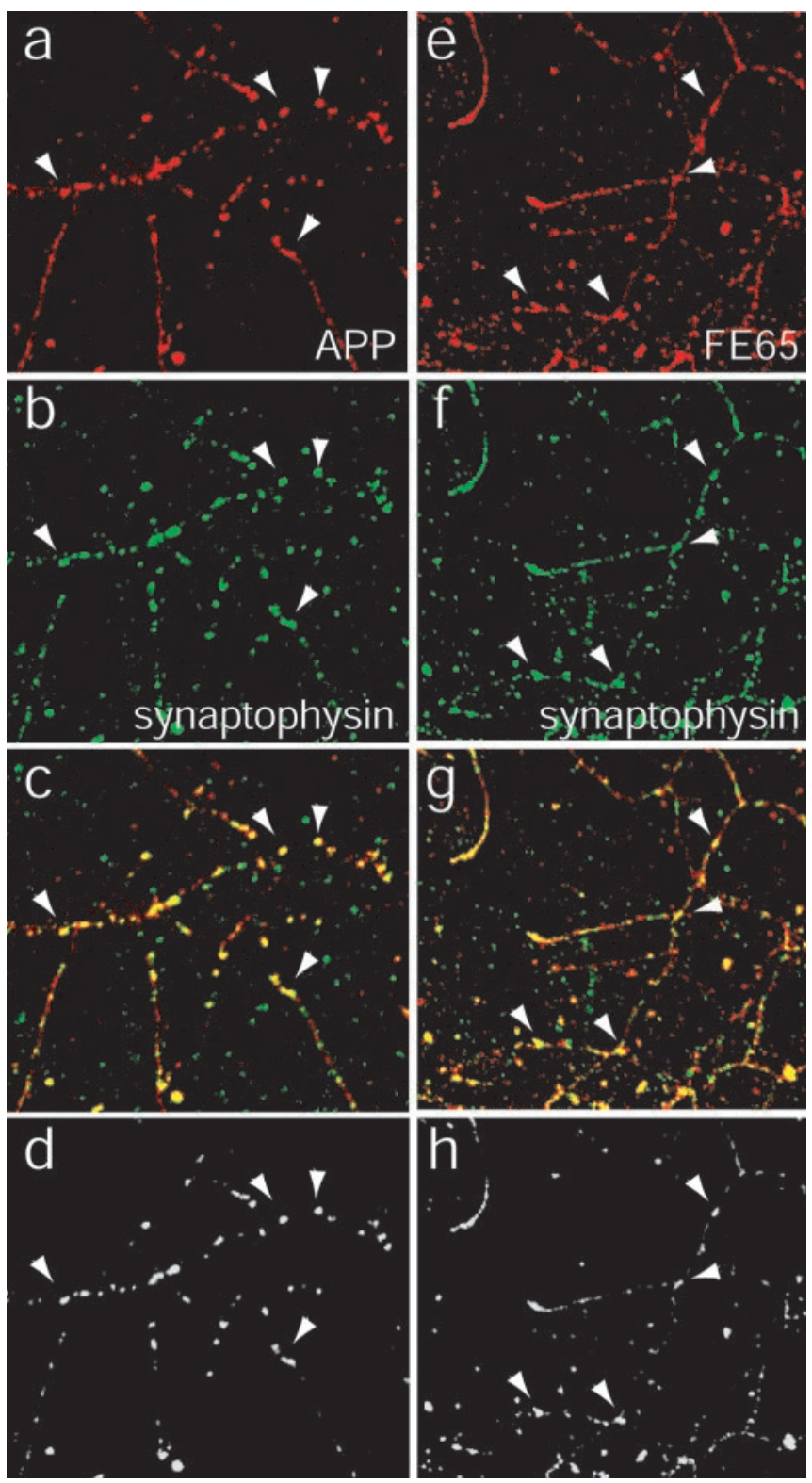

Figure 5. APP and FE65 colocalize with synaptophysin at synapses. $a-c$, Confocal images of the axonal arbors of 7-8 DIV neurons double-labeled with APP ( $a$ ) and synaptophysin ( $b$ ) antibodies. Yellow in the overlay (c) represents colocalization of APP and synaptophysin. Arrows point at a few of the synaptic puncta that contain synaptophysin and APP. $d$, Thresholding and multiplication of the images in $a$ and $b$ yield only the overlapping signal. By comparison with the thresholded synaptophysin signal, this image was used to determine the fraction of synaptophysin-containing sites that also contain APP. $e-g$, Confocal images of the axonal arbors of neurons double-labeled with FE65 $(e)$ and synaptophysin ( $f$ ) antibodies. Yellow in the overlay $(g)$ represents colocalization of FE65 and synaptophysin. Arrows point at a few of the synaptic puncta that contain synaptophysin and FE65. $h$, Thresholding and multiplication of the images in $e$ and $f$ yield only the overlapping signal. By comparison with the thresholded synaptophysin signal, this image was used to determine the fraction of synaptophysin-containing sites that also contain FE65.

synapses from 11 axon segments). Because most neurons in the cultures were labeled by the antibodies, it was difficult to determine with this method whether the APP and FE65 were localized with synaptophysin in the presynaptic terminal or opposite synaptophysin in the postsynaptic terminal. Furthermore, it remained possible that diffuse cytoplasmic staining for FE65 could appear stronger at sites where neurites cross simply because the diffuse signals for the crossing neurites sum at their intersections.
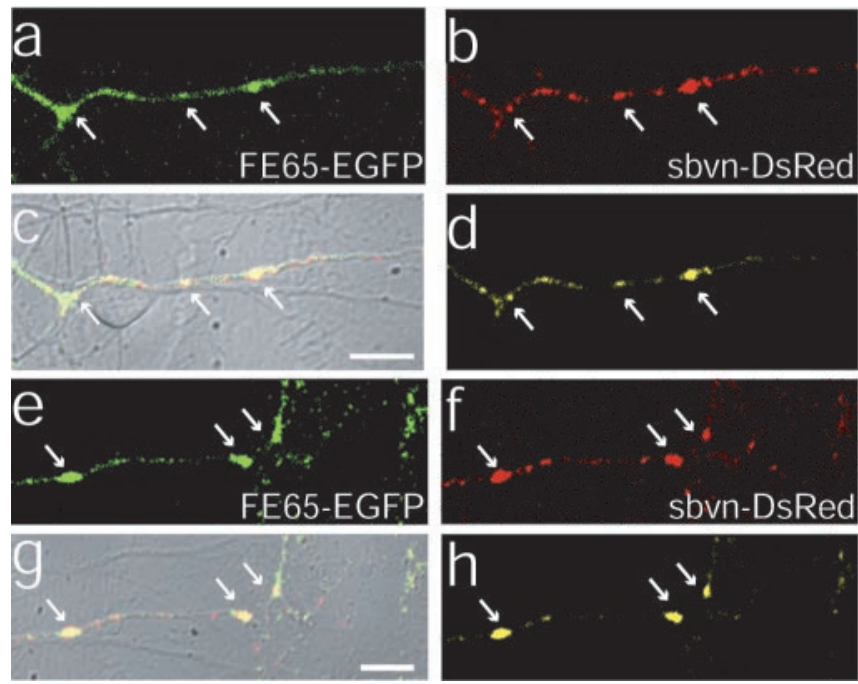

Figure 6. FE65-EGFP colocalizes with synaptobrevin-DsRed in living presynaptic terminals. $a, b, e, f$, Two examples of axon segments of neurons (7-8 DIV) that were double-transfected with FE65-EGFP $(a, e)$ and synaptobrevin-DsRed $(b, f)$ and then examined by live confocal imaging. C, $g$, Overlay of the FE65-EGFP and synaptobrevin-DsRed with the DIC image. Colocalization of the FE65-EGFP and synaptobrevin-DsRed is indicated by yellow in the overlays. Both proteins accumulate along the axon at sites of cell-cell contact, most likely corresponding to synapses. Contact can be seen in the DIC image where the labeled axon is crossed by or touched by an unlabeled neurite or soma. $d, h$, Overlap of FE65-EGFP and synaptobrevinDsRed was extracted by thresholding and multiplying the two signals. The product reveals the sites that contain both proteins. Arrows point to cell-cell contacts that contain both proteins. Scale bars, $10 \mu \mathrm{m}$.

To resolve these issues, 7-8 DIV neurons were cotransfected with FE65-EGFP and synaptobrevin-dsRed cDNAs. The localization of synaptobrevin-dsRed was identical to that of synaptobrevin-EGFP (data not shown), which is correctly targeted to synaptic vesicles (Miesenbock et al., 1998; Nonet, 1999; Ahmari et al., 2000; Estes et al., 2000; Alsina et al., 2001). Furthermore, the labeling pattern looked similar to the pattern seen when endogenous synaptobrevin protein is immunofluorescently labeled. In the overlay of the fluorescence image on the differential interference contrast (DIC) image, FE65 appeared to accumulate at sites of cell-cell contact (Fig. 6a,c,e,g). Sites of contact are defined as portions of labeled axons that are touched or crossed by unlabeled neurites or cell bodies, on the basis of the DIC image. Because these sites of contact contained the synaptic vesicle protein synaptobrevin (Fig. $6 b, c, f, g$ ), they were likely to be synaptic terminals. Because the transfection efficiency was low for these experiments, only one neuron was transfected per field of view; therefore, FE65 that colocalized with synaptobrevin must have been in the presynaptic terminal.

We did not perform a similar analysis of APP-EGFP because it is expected that cytoplasmic domain fusions with GFP would interfere with the interaction of FE65 and APP. However, because the colocalization of APP and FE65 is so strong by immunofluorescence, it seems that we can be confident that the localization of APP and FE65 at presynaptic contacts is likely to be similar. Furthermore, it has been demonstrated previously that APP is localized to presynaptic terminals (Caporaso et al., 1994; Ikin et al., 1996).

In randomly chosen segments of doubly transfected axons $(n=14), 380$ sites of synaptobrevin accumulation were observed; 308 of these synaptobrevin-containing sites also appeared to contain FE65. Within individual axons, $81.0 \pm 2.9 \%$ (mean \pm SE for 14 axons) of presynaptic terminals also contained FE65. This is 

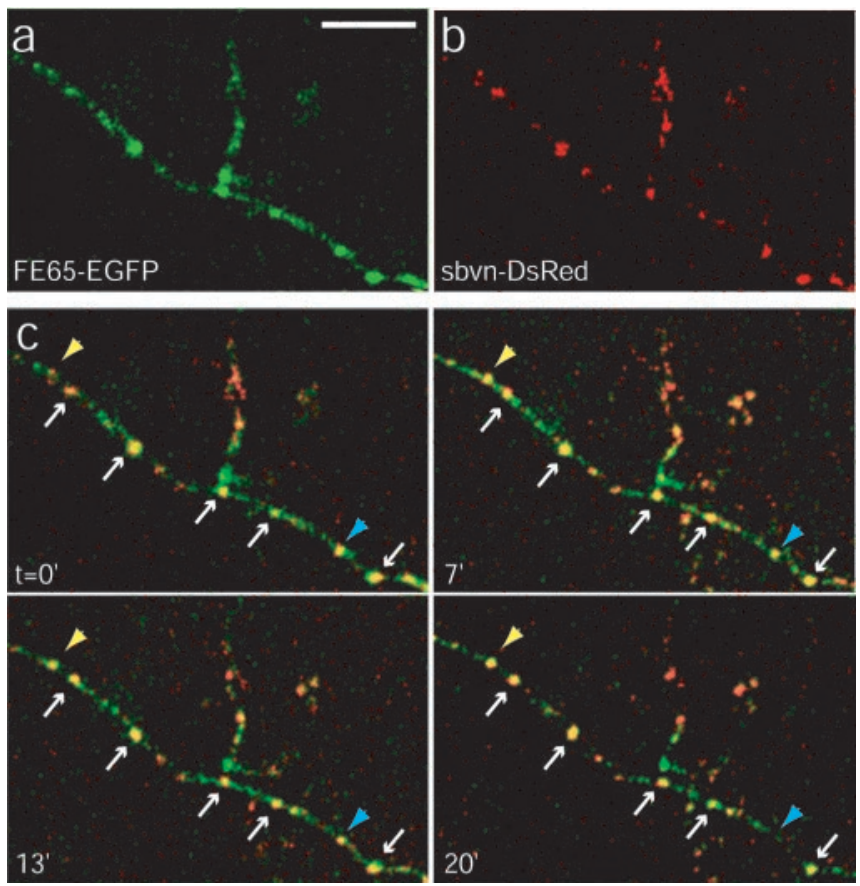

Figure 7. FE65 and synaptobrevin-containing synapses are stable over time. $a-c$, Timelapse confocal microscopy of neurons transfected with FE65-EGFP and synaptobrevin-DsRed. $a$, FE65-EGFP in an axon segment at the start of imaging. $b$, Synaptobrevin-DsRed in the same axon segment at the start of imaging. C, Overlap of FE65-EGFP and synaptobrevin-DsRed signals at the start of imaging $\left(t=0^{\prime}\right)$ and at 7, 13, and 20 min after the start of imaging $\left(7^{\prime}\right.$, $13^{\prime}, 20^{\prime}$, respectively). Arrows point to stationary presynaptic terminals. The blue arrowhead points to a synaptobrevin-containing site that leaves the field of view during imaging (compare $13^{\prime}$ and $20^{\prime}$ ). The yellow arrowhead points to a synaptobrevin-containing site that enters the field of view during imaging (compare $t=0^{\prime}$ and $7^{\prime}$ ) and then remains stable for the duration of imaging. Scale bars, $10 \mu \mathrm{m}$.

not significantly different from the values obtained by immunofluorescence colocalization with synaptophysin $(p>0.05$ by ANOVA), supporting the idea that most of the colocalization seen by immunofluorescence was within the presynaptic terminal.

Of the 126 synaptobrevin and FE65-containing sites in images in which phase-contrast images were also captured, 124 appeared to occur at sites of contact with another neuron and therefore were probable synapses. Focal accumulation of FE65-EGFP was not seen at sites that did not appear to correspond to cell-cell contacts. Moreover, FE65-EGFP fluorescence appeared stronger at sites with a larger contact area. Some diffuse cytoplasmic staining of FE65 was seen throughout many of the neuronal processes, which is not surprising because FE65 is a soluble cytosolic protein. This staining was strongest in proximal regions of the neuronal processes, consistent with the synthesis of FE65 in the cytosol and its diffusion into regions closest to the cell body.

In young neurons, synaptobrevin can be found associated with mobile transport packets that are not synapses (Ahmari et al., 2000). To determine whether the synaptobrevin puncta observed here in older neurons were synapses or transport vesicles, simultaneous time-lapse imaging of FE65-EGFP and synaptobrevin-DsRed was performed. The results revealed that most of the FE65 and synaptobrevin-containing sites were stable for at least $20 \mathrm{~min}$ (Fig. 7). As proof that the neurons were healthy and dynamic, occasionally a site of synaptobrevin accumulation moved into or out of the field of view.

To confirm the presence of FE65 with APP in the presynaptic a

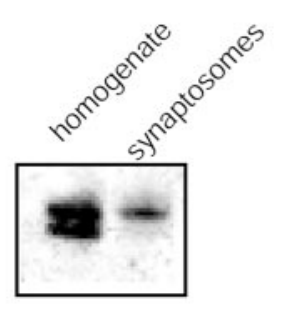

FE65
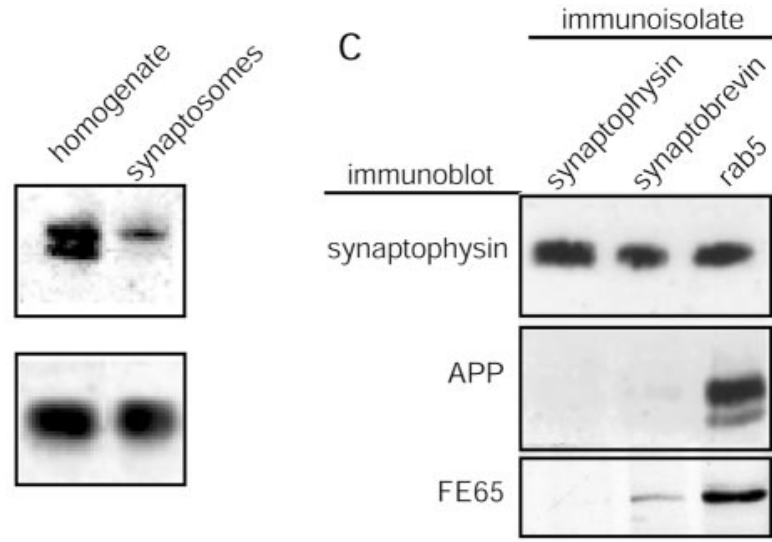

$\mathrm{b}$

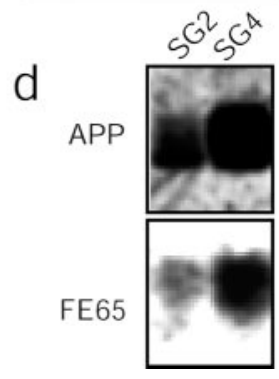

Figure 8. FE65 interacts with APP in nerve terminals. $a$, Synaptosomes probed for FE65 and APP expression by immunoblotting. $b$, Coprecipitation of FE65 from synaptosomes (P2) with APP fused to GST (GST-C50). GST alone (GST) and GST fused to a portion of FE65 (GST-PID) were used as negative controls. c, Synaptic organelles immunoisolated with anti-synaptophysin (synaptophysin), anti-synaptobrevin (synaptobrevin), or anti-rab5 (rab-5) antibodies were immunoblotted for FE65, APP, and synaptophysin. d, Small synaptic vesicles (SG2) were separated from other small synaptic membranes (SG4) by sucrose gradient centrifugation and examined by immunoblotting with FE65 and APP antibodies. The effective higher spatial resolution afforded by the immunoisolation and fractionation approaches, as compared with fluorescence imaging shown in Figures 5 and 6 , shows that APP and FE65 are found in a population of synaptic organelles that are distinct from the small synaptic vesicles that contain synaptophysin and synaptobrevin. PID, Protein interaction domain.

nerve terminal in vivo, synaptosomal fractions were purified from rat brain and immunoblotted with FE65 and APP antibodies. Both FE65 and APP were found in synaptosomes (Fig. 8a), supporting our imaging results. To verify that FE65 from nerve terminals binds to APP, the cytoplasmic domain of APP fused to GST (GST-C50) was immobilized on glutathione-Sepharose beads and then incubated with synaptic membranes (P2). Immunoblotting of bound proteins with FE65 antibodies revealed that synaptic FE65 coprecipitated with GST-C50 but not with GST alone or with an unrelated GST-fusion protein (Fig. $8 b$ ). Thus FE65 from nerve terminals binds to APP.

\section{Like APP, FE65 associates with rab-5-containing organelles} but not with synaptic vesicles

At nerve terminals, APP is localized to Rab5-containing organelles that are distinct from small synaptic vesicles (Ikin et al., 1996). Rab5 is a GTPase required for membrane trafficking between the plasma membrane and early endosomes, both in the soma and at synapses (Bucci et al., 1992; Zerial, 1993; de Hoop et al., 1994; Fischer von Mollard et al., 1994). Fluorescence imaging has insufficient spatial resolution to permit determination of which organelles within a synapse contain FE65; therefore, to test whether FE65 also preferentially associates with the Rab5 organelle, Rab5-containing synaptic organelles were immunoisolated from purified synaptosomes using beads coated with Rab5a antibodies (Fig. 8c). Rab5 immunoisolates contain a significant 
pool of small synaptic vesicles; therefore, synaptophysin and synaptobrevin immunoisolates were prepared in parallel for comparison.

Preferential binding to small synaptic vesicles resulted in nearly equal association with synaptic vesicle protein and Rab5 immunoisolates, as illustrated by immunoblotting for synaptophysin. Conversely, preferential association with the Rab5containing organelles yielded enrichment in the Rab5 fraction, as shown by immunoblotting for APP. Like APP, FE65 was highly enriched in Rab5 immunoisolates compared with synaptophysin or synaptobrevin fractions and therefore was preferentially associated with the Rab5-containing organelle.

To confirm these results, synaptic organelles were separated by sucrose gradient centrifugation and examined by immunoblotting. Fraction 2 of the sucrose gradient (SG2) is enriched in small synaptic vesicles, whereas fraction 4 (SG4) contains other synaptic organelles, such as the Rab5- and APP-containing organelle. Consistent with the immunoisolation results, FE65 and APP were enriched in SG4 compared with SG2 (Fig. 8d). Although FE65 is a cytosolic protein, it cofractionated with membranes that contained APP but not with those that did not, suggesting that FE65 specifically associates with APP-containing synaptic membranes through its interaction with APP.

\section{Discussion}

Here we have shown that FE65 localizes with APP in the peripheral regions of neuronal growth cones, in nerve terminals, and in dendritic filopodia. Dynamic actin plays an important role in each of these subcellular domains, regulating both neuronal development and function. We have shown previously that an APP/ FE65 complex localizes to actin-rich lamellipodia of nonneuronal cells and regulates cell motility (Sabo et al., 2001). Together, our results are consistent with the hypothesis that FE65 and APP regulate actin-based motility in neurons.

FE65 and APP localized to neuronal growth cones both in vitro and in vivo. The $\mathrm{P}$ domains of growth cones are known to be enriched in dynamic actin filaments. These actin filaments are essential for the formation and movement of the growth cone lamellipodia and filopodia. Lamellipodia and filopodia are important for the ability of growth cones to crawl toward their targets and for the growing neurite to sense and respond to its environment.

The localization of FE65 and APP in the growth cone P domain suggests a role for the APP/FE65 complex in regulation of growth cone motility. Effects on growth cone motility are expected to result in altered axon outgrowth. There is evidence in support of APP-dependent regulation of axon growth. For example, APP synthesis and axonal transport coincide with periods of axon elongation and synapse formation (Hung et al., 1992; Moya et al., 1994). In addition, exogenous APP increases neurite outgrowth (Milward et al., 1992; Jin et al., 1994; Small et al., 1994; Perez et al., 1997). Finally, when APP expression is decreased, process outgrowth is altered (LeBlanc et al., 1992; Majocha et al., 1994; Allinquant et al., 1995; Perez et al., 1997). The presence of APP and FE65 in motile regions of growth cones both in vitro and in vivo, our previous data showing that FE65 and APP increase cell motility (Sabo et al., 2001), and the colocalization with Mena, which itself regulates motility (Bear et al., 2000), strongly support a model in which APP and FE65 are involved in the complex regulation of actin-based growth cone motility. Although it is impossible to say from our data whether the interaction of FE65 and APP with either each other or with Mena is responsible for targeting to growth cones, this will be an interesting question to address in the future.

In addition to its localization in growth cones, FE65-EGFP was seen in dendritic filopodia. Dendritic filopodia are thought to be precursors to dendritic spines, which contain the postsynaptic terminals of excitatory synapses. Actin dynamics are believed to provide the structural basis for spine formation and for the rearrangements that are necessary for plasticity of the postsynaptic terminals of mature excitatory synapses (Halpain, 2000; Matus, 2000).

F-actin is essential for the development and maintenance of young synapses (Zhang and Benson, 2001). Moreover, actin is thought to be involved in the formation of new, active presynaptic terminals in response to stimulation, which is important for some forms of long-term facilitation (Hatada et al., 2000; Krucker et al., 2000; Colicos et al., 2001). Within mature synapses, actin appears to localize to the periphery of synaptic vesicle clusters and just outside of the active zone at the endocytic zone (Hirokawa et al., 1989; Cremona and De Camilli, 1997; Brodin et al., 2000; Dunaevsky and Connor, 2000; Morales et al., 2000). The appearance of actin in these regions appears to be coupled to synaptic activity (Brodin et al., 2000).

We showed here that both APP and FE65 localize to presynaptic terminals in vitro and in vivo by immunofluorescence and subcellular fractionation, respectively. Immunofluorescence demonstrated that APP and FE65 localize to synapses that contain synaptophysin. We also confirmed with time-lapse imaging of living neurons that FE65 localizes to synaptobrevin-containing presynaptic terminals. The higher resolution afforded by the fractionation approaches revealed that within synapses APP and FE65 localize to different organelles than synaptophysin. On the basis of the molecular weight of the APP in synaptosome fractions and on our ability to localize APP to nerve terminals by immunofluorescence with either $\mathrm{N}$-terminal or C-terminal antibodies, it is likely that there is a substantial pool of full-length APP that is capable of interacting with FE65 in synapses. We confirmed that FE65 from synapses interacts with APP by performing coprecipitations. Overall, our data are consistent with a role for an APP/FE65 complex in synapses.

We have shown previously that the APP/FE65 complex regulates the motility of non-neuronal cells (Sabo et al., 2001). The function of the APP/FE65 complex in synapses might be related to its role in regulation of membrane motility. A more dynamic neuronal membrane could more easily respond to environmental cues by facilitating morphological change. This might be especially important for dynamic phenomenon, such as synapse formation and synaptic plasticity. Although there is no gross abnormality in the neural development of FE65 knock-out mice (A. F. Ikin and J. D. Buxbaum, unpublished observations), there are two additional FE65 genes (FE65L and FE65L2) that might compensate for loss of FE65. Interestingly, it has been shown that APP knock-out mice exhibit decreased expression of synaptic markers (Dawson et al., 1999) and deficits in synaptic maturation within the cortex (Heber et al., 2000).

Morphological changes, such as those seen during motility, require coordination of cytoskeletal and membrane dynamics. Recent evidence links membrane dynamics important for motility to actin cytoskeletal dynamics through Rab5. Rab5 mediates phorbol ester-induced cell motility and actin cytoskeleton reorganization (Imamura et al., 1998). In addition, active Rab5 induces reorganization of the actin cytoskeleton, lamellipodia formation, and stimulation of cell migration (Spaargaren and Bos, 1999). Interestingly, Rab5 regulates membrane traffic between 
the plasma membrane and early endosomes (Bucci et al., 1992; Zerial, 1993; de Hoop et al., 1994; Fischer von Mollard., 1994), and actin is thought to be important for endocytosis at presynaptic terminals (Cremona and De Camilli, 1997; Brodin et al., 2000). At synapses, Rab5 localizes both to synaptic vesicles and to unique, possibly endocytic, multilamellar organelles (Ikin et al., 1996). APP has been shown previously to localize to these multilamellar organelles (Ikin et al., 1996). We have shown here that APP and FE65, which regulate cell motility, are enriched in the Rab5-containing synaptic organelles that are distinct from synaptic vesicles. Perhaps the Rab5-, APP-, and FE65-containing organelles link cytoskeletal and membrane dynamics in the nerve terminal. The presence of APP and FE65 in these organelles but not in other synaptic membranes implies that the APP/FE65 complex might be involved in this connection.

\section{References}

Ahmari SE, Buchanan J, Smith SJ (2000) Assembly of presynaptic active zones from cytoplasmic transport packets. Nat Neurosci 3:445-451.

Allinquant B, Hantraye P, Mailleux P, Moya K, Bouillot C, Prochiantz A (1995) Down-regulation of amyloid precursor protein inhibits neurite outgrowth in vitro. J Cell Biol 128:919-927.

Alsina B, Vu T, Cohen-Cory S (2001) Visualizing synapse formation in arborizing optic axons in vivo: dynamics and modulation by BDNF. Nat Neurosci 4:1093-1101.

Banker G, Goslin K (1998) Culturing nerve cells. Cambridge, MA: MIT.

Bashaw GJ, Kidd T, Murray D, Pawson T, Goodman CS (2000) Repulsive axon guidance: Abelson and Enabled play opposing roles downstream of the Roundabout receptor. Cell 101:703-715.

Bear JE, Loureiro JJ, Libova I, Fassler R, Wehland J, Gertler FB (2000) Negative regulation of fibroblast motility by Ena/Vasp proteins. Cell 101:717-728.

Brodin L, Low P, Shupliakov O (2000) Sequential steps in clathrinmediated synaptic vesicle endocytosis. Curr Opin Neurobiol 10:312-320.

Bucci C, Parton RG, Mather IH, Stunnenberg H, Simons K, Hoflack B, Zerial M (1992) The small GTPase rab5 functions as a regulatory factor in the early endocytic pathway. Cell 70:715-728.

Caporaso GL, Takei K, Gandy SE, Matteoli M, Mundigl O, Greengard P, De Camilli P (1994) Morphologic and biochemical analysis of the intracellular trafficking of the Alzheimer $\beta /$ A4 amyloid precursor protein. J Neurosci 14:3122-3138.

Chilcote TJ, Galli T, Mundigl O, Edelmann L, McPherson PS, Takei K, De Camilli P (1995) Cellubrevin and synaptobrevins: similar subcellular localization and biochemical properties in PC12 cells. J Cell Biol 129:219-231.

Colicos MA, Collins BE, Sailor MJ, Goda Y (2001) Remodeling of synaptic actin induced by photoconductive stimulation. Cell 107:605-616.

Cremona O, De Camilli P (1997) Synaptic vesicle endocytosis. Curr Opin Neurobiol 7:323-330.

Dawson GR, Seabrook GR, Zheng H, Smith DW, Graham S, O'Dowd G, Bowery BJ, Boyce S, Trumbauer ME, Chen HY, Van der Ploeg LH, Sirinathsinghji DJ (1999) Age-related cognitive deficits, impaired longterm potentiation and reduction in synaptic marker density in mice lacking the beta-amyloid precursor protein. Neuroscience 90:1-13.

de Hoop MJ, Huber LA, Stenmark H, Williamson E, Zerial M, Parton RG, Dotti CG (1994) The involvement of the small GTP-binding protein Rab5a in neuronal endocytosis. Neuron 13:11-22.

Duilio A, Faraonio R, Minopoli G, Zambrano N, Russo T (1998) Fe65L2: a new member of the Fe65 protein family interacting with the intracellular domain of the Alzheimer's beta-amyloid precursor protein. Biochem J 330:513-519.

Dunaevsky A, Connor EA (2000) F-actin is concentrated in nonrelease domains at frog neuromuscular junctions. J Neurosci 20:6007-6012.

Estes PS, Ho GL, Narayanan R, Ramaswami M (2000) Synaptic localization and restricted diffusion of a Drosophila neuronal synaptobrevin-green fluorescent protein chimera in vivo. J Neurogenet 13:233-255.

Ferreira A, Caceres A, Kosik KS (1993) Intraneuronal compartments of the amyloid precursor protein. J Neurosci 13:3112-3123.

Fischer von Mollard G, Stahl B, Walch-Solimena C, Takei K, Daniels L, Khoklatchev A, De Camilli P, Sudhof TC, Jahn R (1994) Localization of
Rab5 to synaptic vesicles identifies endosomal intermediate in synaptic vesicle recycling pathway. Eur J Cell Biol 65:319-326.

Fletcher TL, Cameron P, De Camilli P, Banker G (1991) The distribution of synapsin I and synaptophysin in hippocampal neurons developing in culture. J Neurosci 11:1617-1626.

Gertler FB, Doctor JS, Hoffmann FM (1990) Genetic suppression of mutations in the Drosophila abl proto-oncogene homolog. Science 248:857-860.

Guenette SY, Chen J, Ferland A, Haass C, Capell A, Tanzi RE (1999) hFE65L influences amyloid precursor protein maturation and secretion. J Neurochem 73:985-993.

Halpain S (2000) Actin and the agile spine: how and why do dendritic spines dance? Trends Neurosci 23:141-146.

Hatada Y, Wu F, Sun ZY, Schacher S, Goldberg DJ (2000) Presynaptic morphological changes associated with long-term synaptic facilitation are triggered by actin polymerization at preexisting varicositis. J Neurosci $20: \operatorname{RC} 82(1-4)$.

Heber S, Herms J, Gajic V, Hainfellner J, Aguzzi A, Rülicke T, Kretzschmar H, von Koch C, Sisodia S, Tremml P, Lipp H, Wolfer DP, Müller U (2000) Mice with combined gene knock-outs reveal essential and partially redundant functions of amyloid precursor protein family members. J Neurosci 20:7951-7963.

Hirokawa N, Sobue K, Kanda K, Harada A, Yorifuji H (1989) The cytoskeletal architecture of the presynaptic terminal and molecular structure of synapsin 1. J Cell Biol 108:111-126.

Hung AY, Koo EH, Haass C, Selkoe DL (1992) Increased expression of betaamyloid precursor protein during neuronal differentiation is not accompanied by secretory cleavage. Proc Natl Acad Sci USA 89:9439-9443.

Huttner WB, Schiebler W, Greengard P, De Camilli P (1983) Synapsin I (protein I), a nerve terminal-specific phosphoprotein. III. Its association with synaptic vesicles studied in a highly purified synaptic vesicle preparation. J Cell Biol 96:1374-1388.

Ikin AF, Annaert WG, Takei K, De Camilli P, Jahn R, Greengard P, Buxbaum JD (1996) Alzheimer amyloid protein precursor is localized in nerve terminal preparations to Rab5-containing vesicular organelles distinct from those implicated in the synaptic vesicle pathway. J Biol Chem 271:31783-31786.

Imamura H, Takaishi K, Nakano K, Kodama A, Oishi H, Shiozaki H, Monden M, Sasaki T, Takai Y (1998) Rho and Rab small G proteins coordinately reorganize stress fibers and focal adhesions in MDCK cells. Mol Biol Cell 9:2561-2575.

Jahn R, Schiebler W, Ouimet C, Greengard P (1985) A 38,000-dalton membrane protein (p38) present in synaptic vesicles. Proc Natl Acad Sci USA 82:4137-4141.

Jin LW, Ninomiya H, Roch JM, Schubert D, Masliah E, Otero DA, Saitoh T (1994) Peptides containing the RERMS sequence of amyloid beta/A4 protein precursor bind cell surface and promote neurite extension. J Neurosci 14:5461-5470.

Koo EH, Sisodia SS, Archer DR, Martin LJ, Weidemann A, Beyreuther K, Fischer P, Masters CL, Price DL (1990) Precursor of amyloid protein in Alzheimer disease undergoes fast anterograde axonal transport. Proc Natl Acad Sci USA 87:1561-1565.

Krucker T, Siggins GR, Halpain S (2000) Dynamic actin filaments are required for stable long-term potentiation (LTP) in area CA1 of the hippocampus. Proc Natl Acad Sci USA 97:6856-6861.

Lanier LM, Gates MA, Witke W, Menzies AS, Wehman AM, Macklis JD, Kwiatkowski D, Soriano P, Gertler FB (1999) Mena is required for neurulation and commissure formation. Neuron 22:313-325.

LeBlanc AC, Kovacs DM, Chen HY, Villare F, Tykocinski M, AutilioGambetti L, Gambetti P (1992) Role of amyloid precursor protein (APP): study with antisense transfection of human neuroblastoma cells. J Neurosci Res 31:635-645.

Majocha RE, Agrawal S, Tang JY, Humke EW, Marotta CA (1994) Modulation of the PC12 cell response to nerve growth factor by antisense oligonucleotide to amyloid precursor protein. Cell Mol Neurobiol 14:425-437.

Matus A (2000) Actin-based plasticity in dendritic spines. Science 290:754-758.

McAllister AK, Stevens CF (2000) Non-saturation of both AMPA and NMDA receptors at hippocampal synapses. Proc Natl Acad Sci USA 97:6173-6178.

Miesenbock G, De Angelis DA, Rothman JE (1998) Visualizing secretion 
and synaptic transmission with $\mathrm{pH}$-sensitive green fluorescent proteins. Nature 394:192-195.

Milward EA, Papadopoulos R, Fuller SJ, Moir RD, Small D, Beyreuther K, Masters CL (1992) The amyloid protein precursor of Alzheimer's disease is a mediator of the effects of nerve growth factor on neurite outgrowth. Neuron 9:129-137.

Morales M, Colicos MA, Goda Y (2000) Actin-dependent regulation of neurotransmitter release at central synapses. Neuron 27:539-550.

Moya KL, Benowitz LI, Schneider GE, Allinquant B (1994) The amyloid precursor protein is developmentally regulated and correlated with synaptogenesis. Dev Biol 161:597-603.

Nonet ML (1999) Visualization of synaptic specializations in live C. elegans with synaptic vesicle protein-GFP fusions. J Neurosci Methods 89:33-40.

Perez RG, Zheng H, Van der Ploeg LH, Koo EH (1997) The beta-amyloid precursor protein of Alzheimer's disease enhances neuron viability and modulates neuronal polarity. J Neurosci 17:9407-9414.

Pfenninger KH, Ellis L, Johnson MP, Friedman LB, Somlo S (1983) Nerve growth cones isolated from fetal rat brain: subcellular fractionation and characterization. Cell 35:573-584.

Sabo SL, Lanier LM, Ikin AF, Khorkova O, Sahasrabudhe S, Greengard P, Buxbaum JD (1999) Regulation of beta-amyloid secretion by FE65, an amyloid protein precursor-binding protein. J Biol Chem 274:7952-7957.

Sabo SL, Ikin AF, Buxabum JD, Greengard P (2001) The Alzheimer amyloid precursor protein and FE65, an APP-binding protein, regulate cell movement. J Cell Biol 153:1403-1414.
Shivers BD, Hilbich C, Multhaup G, Salbaum M, Beyreuther K, Seeburg PH (1988) Alzheimer's disease amyloidogenic glycoprotein: expression pattern in rat brain suggests a role in cell contact. EMBO J 7:1365-1370.

Small DH, Nurcombe V, Reed G, Clarris H, Moir R, Beyreuther K, Masters CL (1994) A heparin-binding domain in the amyloid protein precursor of Alzheimer's disease is involved in the regulation of neurite outgrowth. J Neurosci 14:2117-2127.

Spaargaren M, Bos JL (1999) Rab5 induces Rac-independent lamellipodia formation and cell migration. Mol Biol Cell 10:3239-3250.

Tanahashi H, Tabira T (1999) Molecular cloning of human Fe65L2 and its interaction with the Alzheimer's beta-amyloid precursor protein. Neurosci Lett 261:143-146.

Torroja L, Packard M, Gorczyca M, White K, Budnik V (1999) The Drosophila beta-amyloid precursor protein homolog promotes synapse differentiation at the neuromuscular junction. J Neurosci 19:7793-7803.

Yamazaki T, Selkoe DJ, Koo EH (1995) Trafficking of cell surface betaamyloid precursor protein: retrograde and transcytotic transport in cultured neurons. J Cell Biol 129:431-442.

Yamazaki T, Koo EH, Selkoe DJ (1997) Cell surface amyloid beta-protein precursor colocalizes with beta 1 integrins at substrate contact sites in neural cells. J Neurosci 17:1004-1010.

Zerial M (1993) Regulation of endocytosis by the small GTP-ase rab5. Cytotechnology 11[Suppl 1]:S47-49.

Zhang W, Benson DL (2001) Stages of synapse development defined by dependence on F-actin. J Neurosci 21:5169-5181. 